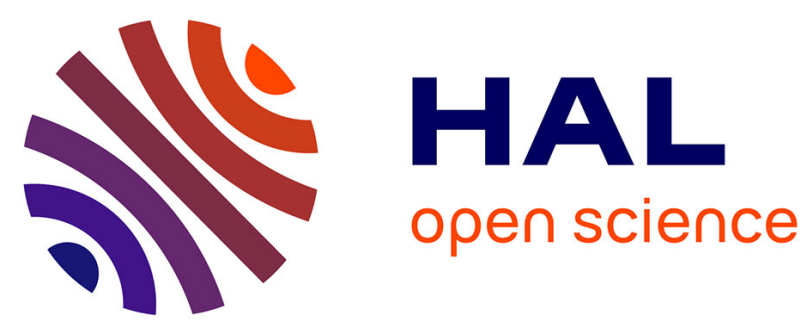

\title{
L'éducation thérapeutique: un levier pour modifier les perceptions du trouble bipolaire chez les aidants familiaux
}

\author{
K. M’bailara, I. Minois, L. Zanouy, F. Josse, E. Rouan, A. Maîtrot, J. \\ Sportich, S. Roux, A. Jutant, A. Deloge, et al.
}

\section{To cite this version:}

K. M'bailara, I. Minois, L. Zanouy, F. Josse, E. Rouan, et al.. L'éducation thérapeutique: un levier pour modifier les perceptions du trouble bipolaire chez les aidants familiaux. L'Encéphale, 2019, 45, pp.239 - 244. 10.1016/j.encep.2018.11.004 . hal-03486812

\section{HAL Id: hal-03486812 \\ https://hal.science/hal-03486812}

Submitted on 20 Dec 2021

HAL is a multi-disciplinary open access archive for the deposit and dissemination of scientific research documents, whether they are published or not. The documents may come from teaching and research institutions in France or abroad, or from public or private research centers.
L'archive ouverte pluridisciplinaire HAL, est destinée au dépôt et à la diffusion de documents scientifiques de niveau recherche, publiés ou non, émanant des établissements d'enseignement et de recherche français ou étrangers, des laboratoires publics ou privés.

\section{(c) (1) $\$$}

Distributed under a Creative Commons Attribution - NonCommerciall 4.0 International 


\section{L'Education Thérapeutique : un levier pour modifier les perceptions du trouble bipolaire chez les aidants familiaux}

Therapeutic Education: A lever to change perceptions of bipolar disorder in family caregivers

\section{K. M'Bailara ${ }^{(1,2,3)}$, I. Minois ${ }^{(2,3)}$, L. Zanouy ${ }^{(2,3)}$, F. Josse ${ }^{(2,3)}$, E. Rouan ${ }^{(4),}$ A. Maîtrot ${ }^{(5)}$, J. Sportich ${ }^{(2,3)}$, S. Roux ${ }^{(1)}$, A. Jutant ${ }^{(2)}$, A. Deloge ${ }^{(2)}$, A. Desage(2) et S. Gard(2,3),}

(1) Laboratoire de psychologie, EA4139, Université de Bordeaux

${ }^{\text {(2) }}$ Centre Hospitalier Charles Perrens, Pôle 3-4-7, Bordeaux

${ }^{(3)}$ Centre Expert Trouble bipolaire, Fondation FondaMental, Fondation de Coopération Scientifique, Créteil

(4) Cabinet libéral, Saucat

${ }^{(5)}$ Cabinet libéral, Bordeaux

Correspondig author:

katia.mbailara@u-bordeaux.fr

Les auteurs déclarent ne pas avoir de liens d'intérêt. 


\title{
L'Éducation Thérapeutique : un levier pour modifier les perceptions du trouble bipolaire chez les aidants familiaux
}

\author{
Therapeutic Éducation: A lever to change perceptions of bipolar \\ disorder in family caregivers
}

\section{Résumé}

Le patient et sa famille sont pris dans une interaction réciproque : dans un sens la pathologie entraîne des souffrances familiales et dans l'autre, le comportement familial retentit sur la maladie du patient et son évolution. Il semble donc essentiel pour le clinicien de travailler avec la famille sur le rapport qu'elle entretient avec le trouble bipolaire, c'est-à-dire ses connaissances mais surtout ses représentations de la pathologie. Notre étude vise à observer si le rapport initial au trouble bipolaire évolue après le suivi d'un programme d'ETP. Menée au Centre Expert Bipolaire de Bordeaux, notre recherche s'appuie sur un échantillon de 145 participants (78 patients et 67 accompagnants familiaux), tous questionnés avant et après le programme d'ETP (12 séances sur 6 mois). Le Brief Illness Perception Questionnaire revised (Moss-Morris, 2002) évalue la perception du trouble bipolaire, l'IPQ-R permet d'obtenir un score évaluant la menace perçue générée par la maladie, tandis que le BP Quizz (Fondation Fondamental) enregistre le niveau de connaissance sur le trouble. Les résultats montrent une amélioration des connaissances et une modification des représentations du trouble. Les aidants, au même titre que les patients, perçoivent ainsi le trouble bipolaire comme moins menaçant, moins sévère et plus contrôlable. En éducation thérapeutique, la juste posture du clinicien serait alors de soutenir ce processus d'évolution et le changement des représentations, au-delà de transmettre des connaissances sur le trouble.

Mots-clés : éducation thérapeutique; trouble bipolaire; famille; perception ; fonctionnement psychosocial

\section{Abstract}

Objectives: The patient with bipolar disorder and his family are caught in a reciprocal interaction: on the one hand, the pathology leads to family sufferings and on the other hand, family behavior affects the disease of the patient and its development. Therefore, it seems of core importance that the psychologist should work with the family on their perception of bipolar disorder, that is to say, on their knowledge and psychological representations of the pathology. The aim of our study is to assess whether the initial perception of bipolar disorder evolves after a therapeutic education program.

Method: Our research was conducted at the Bipolar Expertise Centre in Bordeaux / Centre Expert Bipolaire in Bordeaux on a sample of 145 participants (78patients and 67 
family caregivers). They were all interviewed before and after the Therapeutic Education program (12 sessions in 6 months). The Brief Illness Perception Questionnaire revised (Moss-Morris, 2002) measures the perception of bipolar disorder and the BP Quizz (Fondation Fondamental) assesses the degree of knowledge of the disorder. Results: Results show that Therapeutic Education helps families to level up their knowledge about bipolar disorder. Furthermore, representations on bipolar disorder have globally changed so that on average, bipolar disorder is viewed as less threatening by families after 12 sessions of Therapeutic Education. More precisely, after the program families have a better understanding and a better insight of the disorder which is then perceived as being less severe. On the emotional level, anxiety and stress have decreased. So there are an increase of knowledge and a change in perception. Conclusion: Our study shows that PTE enables families to change their perception of the disease, that is to say, their knowledge but also their representations of the disease, which is a fundamental element according to the models of Therapeutic Education. Our results point out one of the active processes of Therapeutic Education at work in the sessions: in the perception of the disease, which is composed of both knowledge and representations of the disease, just a change in representations constitutes a lever for Therapeutic Education. Therefore, working on representations should be a therapeutic target. As a conclusion, we can say that Therapeutic Education of families cannot be reduced to an educational dimension which would only consist of gaining knowledge. Then, the right posture of the psychologist is to hold each participant's own development and changing process of representations.

Keywords: therapeutic education; bipolar disorder; family; representation ; psychosocial functioning

\section{INTRODUCTION}

Les troubles bipolaires sont une pathologie invalidante puisque classée sixièmecause de handicap par l'organisation mondiale de la santé : 1,5\% de la population en est affectée pour les formes les plus sévères (mais probablement près de $5 \%$ si l'on considère les maladies apparentées) et $15 \%$ des patients décèdent encore à l'heure actuelle par suicide [1]. Ils se caractérisent par l'alternance de phases de dépression et de phases de manie ou hypomanie, le tout entrecoupé de phases de normothymie [2;3]. Reconnus comme maladie chronique, leur niveau élevé de handicap s'exprime tant par une désinsertion sociale, que professionnelle ou familiale [4;5]. Le coût de cette pathologie est considérable. Il est supporté à différents niveaux, tout d'abord par le patient en termes de souffrance et d'exclusion sociale, par l'entourage du patient en termes de fardeau mais aussi plus largement par la société en termes de dépenses de santé et de réhabilitation sociale.

Les troubles bipolaires s'accompagnent de dommages tant objectifs que subjectifs [6]. Les premiers concernent la gestion du quotidien, les tensions intra-familiales, la réduction des relations sociales, le ralentissement d'évolution de carrière, les problèmes financiers et les conséquences sur les enfants. Les seconds concernent le stress plus ou moins chronique par peur des rechutes ou des troubles du comportement. Les dommages générés par la pathologie envahissent donc le quotidien du patient mais aussi de son entourage. L'attitude de la famille peut en retour avoir des conséquences sur le déclenchement et l'évolution de la pathologie. Pour exemple, le niveau d'émotion 
exprimée est un puissant prédicteur de rechutes et d'évolution négative du trouble $[7 ; 8]$. Les émotions exprimées mesurent la manière dont un membre de la famille s'exprime en des termes critiques ou en montrant des signes de surimplication émotionnelle à l'égard d'un autre membre souffrant d'un syndrome psychiatrique [9]. Les familles de patients bipolaires ont un haut niveau d'expressivité émotionnelle qui se manifeste par un surinvestissement émotionnel, de l'hostilité ou des critiques [10], un niveau élevé de conflit et un bas niveau de cohésion et d'expression de soi [11]. Le trouble et ses manifestations ont donc une influence considérable sur la famille et la famille en retour peut avoir une influence sur l'évolution du trouble. Le constat de cette interdépendance incite à conseiller une implication adaptée de la famille dans la prise en charge.

Parmi les prises en charge recommandées dans les troubles bipolaires, figure la psychoéducation [12]. Existant depuis longtemps dans le champ de la santé mentale, la psychoéducation a une visée très proche de l'Éducation Thérapeutique, largement développée pour les maladies chroniques somatiques, et dont la démarche est émergente en psychiatrie. Selon l'OMS [13], l'éducation thérapeutique (ETP) vise à aider les patients à acquérir ou maintenir les compétences dont ils ont besoin pour gérer au mieux leur vie avec une maladie chronique. Les objectifs sont d'une part l'acquisition et le maintien de compétences d'autosoins (à savoir les décisions en faveur de la modification des effets de la maladie sur la santé) [14] et d'autre part, la mobilisation ou l'acquisition de compétences d'adaptation psychosociales. Ces dernières regroupent des compétences personnelles et interpersonnelles, cognitives et physiques qui permettent aux personnes d'acquérir la capacité à vivre dans leur environnement et à le modifier [15].

Bien que la littérature internationale ne fournisse pas de données scientifiques sur l'intérêt de l'Éducation Thérapeutique dans les troubles bipolaires, elle a toutefois montré qu'en psychoéducation les patients acquièrent des compétences que la démarche en éducation thérapeutique qualifierait d'auto-soin [16]. Il en résulte une diminution du risque de rechute et une réduction de la sévérité avec des rechutes moins intenses et moins longues $[17 ; 18 ; 19 ; 20 ; 21]$. La psychoéducation conduit aussi à moins d'hospitalisation après 2 ans [19] et permet un maintien de l'effet à 5 ans [20], avec un effet sur les phases maniaques plus clair que sur les phases dépressives [22]. Enfin, elle améliore l'observance médicamenteuse [23; 24]. Participer à de tels programmes est efficace tant pour les patients ayant un trouble bipolaire de type 1 [25], que de type 2 [26], que pour ceux ayant une comorbidité de trouble de personnalité [27]. Concernant la psychoéducation proposée aux familles de patients ; seules les équipes de D'Souza [21] et de Miller [28] proposent les résultats d'essais thérapeutiques qui incluent les patients et les familles dans leurs groupes, montrant ainsi un effet sur l'évolution du trouble du patient. Les autres études incluent uniquement des familles $[29 ; 30 ; 31 ; 32 ; 33]$ avec les travaux des équipes de Reinares [34;32;35] et ceux de Miklowitz et Goldstein [36; 37; 10; 7; 38; 39] faisant ici école. Les sessions proposent des thèmes sur le trouble bipolaire, son traitement et la gestion du stress et autres risques pour la santé. Ces programmes transmettent des conseils pratiques [40] essentiellement centrés sur la prévention des rechutes. Les résultats sont en faveur de l'amélioration des relations intrafamiliales et l'acquisition de stratégies centrées sur le problème [40], compétences que la démarche en éducation thérapeutique qualifierait d'adaptation psychosociale. Néanmoins, il existe encore peu de littérature sur l'efficacité des 
programmes à visée des familles, ce qui peut en partie expliquer pourquoi il y a si peu d'implémentation d'Éducation Thérapeutique pour ce public en pratique courante.

Pour questionner la pertinence de l'implication des familles, il convient de débuter par l'évaluation de leur rapport au trouble bipolaire. Par "rapport à la maladie», nous entendons le niveau de connaissances mais aussi les représentations des familles sur la pathologie bipolaire. Ce travail sur les représentations est en effet une des cibles du travail en Éducation Thérapeutique [41]. La représentation correspond à un "état de connaissance antérieure, un apprentissage systématique [...], une forme de connaissance pratique" [41]. Dans le cas d'un trouble bipolaire, les représentations des familles peuvent concerner l'origine et les facteurs étiopathogéniques, les symptômes, l'évolution de la pathologie mais également le contrôle qu'aurait potentiellement le patient sur sa propre pathologie. Or à ce jour trop peu de travaux en santé mentale s'intéressent aux représentations des familles [42], alors même que le patient baigne dans ces représentations et que sa vie est impactée par celles-ci.

En synthèse, bien que la littérature concernant les prises en charge des familles de patients ayant un trouble bipolaire en soit à ses balbutiements, il devient nécessaire de soutenir et d'accompagner les patients mais aussi leurs familles dans leur processus d'ajustement au trouble [43]. La HAS conseille aux patients de consulter lorsque lui ou son entourage constate une modification de son humeur [44]. Ce point renvoie à la nécessité pour les familles d'avoir compris la pathologie et de savoir la repérer. L'Éducation Thérapeutique apparaît donc comme une indication non seulement pertinente mais quasi indispensable. Il s'agit de savoir répondre aux besoins sans pour autant étouffer, tout en accompagnant l'autonomisation pour reprendre ici la notion de compétences des familles chère à Ausloos [45].

Cette étude est née du constat d'un paradoxe : les familles sont davantage impliquées dans le soin, mais sans capacité d'adaptation à la pathologie elles risquent de se sentir démunies [46]. L'objectif de cette étude est d'évaluer les modifications du rapport au trouble bipolaire chez des familles incluses dans une prise en charge de groupe en Éducation Thérapeutique. Nous émettons l'hypothèse que les aidants familiaux vont avoir une représentation de la maladie moins menaçante après les séances d'ETP par rapport à leur représentation avant les séances.

\section{MÉTHODOLOGIE}

\section{Procédure}

Cette recherche a été menée au Centre Expert Bipolaire de Bordeaux, au sein du Centre Hospitalier Charles Perrens. Ce Centre Expert des troubles bipolaires, inséré dans le réseau des centres experts nationaux soutenus par la Fondation FondaMental, a pour objectif de favoriser le dépistage et le diagnostic précoce, d'offrir un bilan diagnostic complet (psychiatrique, somatique, psychologique et neuropsychologique) réalisé par une équipe pluridisciplinaire spécialisée dans cette pathologie et enfin, d'améliorer les pratiques et la formation grâce à une constante interaction avec la recherche. Par ailleurs, ces centres ont pour mission de développer et de déployer des pratiques innovantes.

Le programme d'Éducation Thérapeutique est composé de 12 séances de 90 minutes réparties sur 6 mois dispensées en groupe fermé. Il est composé de patients et de familles. Est incluse dans le groupe famille toute personne «telle qu'elle se présente » en 
tant que famille, en référence à la définition de travail proposée par Favez [47]. Le recueil des données s'effectue à 2 reprises : la première visite (V1) a lieu dans les 15 jours qui précèdent la première séance et la deuxième visite (V2) a lieu dans les 15 jours qui suivent la douzième séance.

\section{Population}

L'échantillon est composé de patients et d'aidants familiaux ayant participé à des groupes d'Éducation Thérapeutique multifamiliaux. Tous sont aidants d'un patient ayant reçu le diagnostic de trouble bipolaire selon les critères du DSM-5 [2].

\section{Outils}

Le Brief Illness Perception Questionnaire Revised [48] évalue la perception du trouble bipolaire. Il comprend 8 items cotés sur une échelle à 10 points de Likert où $1=$ pas du tout d'accord et 10 = tout à fait d'accord. Le questionnaire IPQ-R (Illness Perception Questionnaire Revised) original a été validé pour ce type d'étude grâce à l'attestation de ses propriétés psychométriques (validité interne, reproductibilité, pouvoir discriminant, valeur prédictive) sur sept groupes de patients atteints d'une maladie chronique (incluant des patients asthmatiques, diabétiques et hémodialysés). Il permet d'obtenir un score exprimant la menace générée par la maladie qui servira de base à l'évaluation de l'évolution de la représentation de la maladie avant et après le programme d'éducation thérapeutique. Plus le score est élevé plus la menace perçue à l'égard de la maladie est élevée. Le score minimal est de 8, le score maximal possible est de 80. Les items évaluent : la conscience du trouble, la perception de chronicité, le manque de contrôle, la perception de sévérité du trouble, l'intensité de l'inquiétude, la perception d'incompréhension, l'impact émotionnel et l'accord avec le diagnostic. Les Alpha de Cronbach sont de 0.57 avant la participation au programme d'éducation thérapeutique et 0.72 après.

Le Quizz-BP (Fondation FondaMental) évalue le niveau de connaissances sur le trouble bipolaire au moyen de 20 questions en vrai ou faux (occurrence, étiologie, sémiologie, facteurs déclenchants d'épisodes, comorbidités, traitement). L'intervalle de ce score s'étend donc de 0 à 20.

\section{RÉSULTATS}

L'échantillon est composé de 145 personnes (cf tableau 1). Toutes sont soit des aidants naturels de patients ayant un trouble bipolaire, soit des patients. La moyenne d'âge est de 46.39ans (SD12.2) min 20 et max 79). Pour les aidants cette moyenne d'âge est de 48.5 ans (SD 12.5), et pour les patients la moyenne d'âge est de 44.6 ans (SD 11.7). Il y a 51 hommes (35.2\%) et 94 femmes (64.8\%). Pour les aidants il y a 41 femmes $(61.2 \%)$ pour 26 hommes (38.8\%) et pour les patients 53 femmes (67.9\%) pour 25 hommes (32.0 \%). Il n'y a pas de différence significative de la répartition hommes/femmes selon qu'il s'agisse des aidants ou des patients : ? $2(1, \mathrm{~N}=145)=0.45, \mathrm{p}=0.4998$. C'est également le cas pour le niveau d'études. Il n'y a pas de différence significative entre les aidants et les patients concernant le niveau d'études : ? $2(1, N=145)=0.15, p=0.6951$. 
Après avoir participé à un groupe multifamilial d'Éducation Thérapeutique les aidants ont une meilleure connaissance du trouble bipolaire. Il y a une différence significative entre l'évaluation qui a lieu juste avant le groupe (T1) (m=11.84, SD=4.26) et l'évaluation juste après la dernière séance $(\mathrm{T} 2)(\mathrm{m}=15.72, \mathrm{SD}=3.07)$ sur la connaissance avec une augmentation de celle-ci à T2. ( $\mathrm{V}=4889$, p-value $=3.932 \mathrm{e}-14$ ) (cf tableau 2).

Après avoir participé à un groupe multifamilial d'Éducation Thérapeutique les patients ont une meilleure connaissance du trouble bipolaire. Il y a une différence significative entre $\mathrm{T} 1(\mathrm{~m}=12.34, \mathrm{SD}=4.46)$ et $\mathrm{T} 2(\mathrm{~m}=15.7, \mathrm{SD}=3.42)$ sur la connaissance avec une augmentation de celle-ci à T2. $(\mathrm{V}=1917.5$, p-value $=3.21 \mathrm{e}-08)$ (cf tableau 3$)$.

\section{Modification de la perception de la maladie}

Il y a une différence significative entre $\mathrm{T} 1(\mathrm{~m}=50.92, \mathrm{SD}=10.05$; [26-78]) et T2 ( $\mathrm{m}=41.1$, $\mathrm{SD}=13.45$; [1-72]) sur la perception globale avec une diminution du score à $\mathrm{T} 2 . \mathrm{V}=757.5$, $\mathrm{p}$-value $=4.64 \mathrm{e}-12$. Le trouble bipolaire est perçu comme moins menaçant par les aidants après la participation à un groupe d'Éducation Thérapeutique qu'avant la participation. Les aidants ont, en particulier une meilleure conscience du trouble et un meilleur accord avec le diagnostic. Ils perçoivent les symptômes de leur poche comme moins sévères. La chronicité du trouble est perçue comme moins menaçante, ils rapportent moins d'inquiétude en rapport avec le trouble, moins d'incompréhension. Enfin, l'impact émotionnel associé au trouble diminue. Cependant il n'y a pas d'évolution des scores de manque de contrôle et de doute sur le traitement (cf tableau 2).

Après avoir participé au groupe les patients ont en moyenne une perception moins menaçante du trouble. En particulier, les patients comprennent mieux leur trouble et sont davantage en accord avec le diagnostic. Ils estiment avoir un meilleur contrôle sur le trouble et pensent que le traitement peut les aider à faire face à leur trouble. Ils s'inquiètent moins au sujet de leur trouble après la participation qu'avant et se disent moins affectés émotionnellement. Cependant il n'y a pas d'évolution des scores de conscience, de chronicité et de sévérité. Il est à noter des effets plafond et plancher pour les 2 derniers scores. En effet, avant la participation les patients estiment que le trouble est chronique ce qui perdure après le groupe et constitue une réalité au sens ou la vulnérabilité à déclencher des épisodes maniaques ou dépressifs est chronique et constitue la définition même du trouble. Au niveau des symptômes les patients rapportent avoir peu de symptômes avant comme après le groupe ce qui est en accord avec les critères d'inclusion dans le groupe (cf tableau 3).

\section{DISCUSSION}

L'objectif de cette étude était d'évaluer les modifications de perception du trouble bipolaire chez des familles suite à la prise en charge groupale de patients en Éducation Thérapeutique. Les résultats montrent que les aidants familiaux ont une meilleure connaissance sur le trouble bipolaire après avoir participé à un groupe d'Éducation Thérapeutique, mais surtout que leur perception de la maladie se modifie. En effet le trouble bipolaire est alors perçu comme moins menaçant en particulier grâce à une amélioration de la compréhension et de la conscience du trouble. L'impact émotionnel associé au trouble est donc moindre et l'inquiétude diminue. Par ailleurs les aidants familiaux perçoivent le trouble comme moins sévère et rapportent un sentiment de plus grand contrôle. L'ensemble de ces éléments évoque un changement de rapport à la 
maladie, c'est-à-dire des connaissances mais aussi des représentations sur le trouble bipolaire, élément de base selon les modèles en Éducation Thérapeutique. Les patients qui récupèrent un sentiment de contrôle face au trouble, sont davantage en accord avec le diagnostic et ont moins de doute concernant la nécessité d'avoir un traitement. L'intensité de leur inquiétude diminue. Ils se sentent moins dans l'incompréhension et l'impact émotionnel est considéré comme moindre. Du côté des familles, le niveau de conscience de la maladie est meilleur tout comme leur degré d'accord avec le diagnostic. Elles perçoivent ce trouble comme étant moins sévère. Les aidants familiaux se disent être moins dans l'incompréhension face au trouble bipolaire de leur proche. Leur niveau d'inquiétude diminue et l'impact émotionnel associé au trouble est moindre.

Si l'effet positif de l'Éducation Thérapeutique sur l'auto-soin est largement démontré, les effets sur les compétences d'adaptation psychosociale restent moins documentés par la littérature [49]. En effet, jusqu'alors les études avaient surtout montré l'intérêt de l'Éducation Thérapeutique concernant les prises de décisions du patient modifiant l'effet du trouble bipolaire sur sa santé $[16 ; 17 ; 18 ; 19 ; 20 ; 21 ; 22 ; 23 ; 24]$. Une des plusvalues de notre étude est de montrer que les participants ont une perception moins menaçante du trouble après avoir participé au groupe, ce qui pourrait favoriser l'acquisition de compétences d'adaptation psychosociales, seconde cible de l'Éducation Thérapeutique. Ce qu'apporte notre étude n'est pas tant du côté de la quantification des effets que de la mise en évidence du processus en jeu pendant des séances d'Éducation Thérapeutique. Le travail autour des représentations est une cible thérapeutique au cours des ateliers. Ainsi, nos résultats s'inscrivent dans la lignée de travaux récents effectués auprès de patients qui montrent que seul le changement de perception de la maladie est associé au fonctionnement alors que la modification des connaissances n'impacte pas le fonctionnement psychosocial [24]. L'Éducation Thérapeutique des patients mais aussi des familles favorise l'acquisition de compétences d'adaptation psychosociale et ne peut être réduite à une dimension pédagogique qui consisterait uniquement en l'acquisition de connaissances. Au regard de la loi HSPST, «L'Éducation Thérapeutique s'inscrit dans le parcours de soins du patient. Elle a pour objectif de rendre le patient plus autonome en facilitant son adhésion aux traitements prescrits et en améliorant sa qualité de vie» [50]. Nos résultats confirment que l'Éducation Thérapeutique ne peut se réduire à la dimension d'instruction et doit être envisagée comme une thérapeutique à part entière avec son cadre et ses finalités [51]. Ce message est essentiel pour la pratique clinique car il situe d'emblée la posture du clinicien. En Éducation Thérapeutique le clinicien n'est pas seulement du côté de la transmission de l'information, dans une posture qui serait celle l'enseignant possédant le savoir mais bien du côté de l'éducation au sens d'un accompagnement vers un changement personnalisé. Il s'agit de partir du savoir et de la représentation du patient pour l'aider à cheminer vers un changement respectant sa subjectivité tout en l'aidant à développer de nouvelles ressources.

Notre étude présente aussi un enjeu concernant la place des familles en psychiatrie. Dans la littérature il existe assez peu de données sur les familles [52], encore moins sur l'Éducation Thérapeutique multifamiliale qui inclut dans un même dispositif un regroupement de plusieurs patients et de leur famille. Concernant l'impact fonctionnel sur la famille, la littérature est quasi désertique. La littérature ne renseigne pas sur les effets que pourrait avoir la psychoéducation sur les familles elles-mêmes, prenant par exemple en compte le niveau de fardeau, la qualité de vie ou le fonctionnement 
psychosocial. Dans la littérature internationale, les études montrent que la psychoéducation familiale participe à l'amélioration de la communication intrafamiliale [53], à l'amélioration du niveau de fonctionnement [17] et de l'ajustement familial [54], à une meilleure connaissance de la pathologie et une meilleure qualité de soutien [55]. L'évolution de la pathologie est par ailleurs favorablement impactée chez les patients lorsque la famille a pu bénéficier des séances d'Éducation Thérapeutique. On repère ainsi une meilleure prévention des rechutes, une diminution de la sévérité des décompensations et une augmentation de l'observance [56]. Le travail autour de la place des familles est encore long mais semble, au vu de nos résultats un enjeu pour les soins en psychiatrie. En effet, il est intéressant de voir la perception globale se modifier pour les patients mais aussi pour les familles bien qu'il ne s'agisse pas forcément des mêmes dimensions de la perception.

Une des perspectives serait de faire des analyses dyadiques qui pourraient prendre en compte la famille comme entité clinique afin de mieux saisir les modifications au niveau de la dynamique familiale (interactions) et pas uniquement en observant d'un côté les patients et de l'autre sa famille. Notre étude comporte plusieurs limites. La première, d'importance majeure, concerne l'absence de groupe contrôle. Une des perspectives serait de proposer une étude avec un design de type essai contrôlée randomisé afin de répondre aux exigences scientifiques des études d'efficacité des prises en charge thérapeutiques. Nos résultats sont peut-être l'effet d'une évolution normale d'ajustement à la pathologie, n'étant pas obligatoirement lié au groupe. Une autre limite concerne l'hétérogénéité des familles et donc des groupes. Cependant cette étude a pour intérêt de rendre compte de la clinique en pratique courante, à savoir telle qu'elle existe dans les services de psychiatrie français et ne répond effectivement pas au standard d'études de type essai contrôlé randomisé. Enfin, concernant le nombre d'évaluations, la modification de perception a été évaluée en effectuant un delta entre avant et après les sessions d'ETP, il serait donc intéressant de poursuivre l'étude avec des mesures à plus long terme.

\section{BIBLIOGRAPHIE}

[1] World Health Organization. Skills for Health. Geneva: WHO; 2003

[2] American Psychiatric Association. DSM-5 Diagnostic and statistical manual of mental disorders. 2013. Arlington: American Psychiatric Publishing.

[3] Crocq MA, Guelfi JD, Boyer P, et al. Mini DSM-5 Critères Diagnostiques. 2016. Elsevier Health Sciences

[4] Shastry BS. Bipolar disorder: an update. Neurochem Int. 2005;Mar;46(4):273-9. R

[5] Grande I, Berk M, Birmaher B, et al. Bipolar disorder. Lancet. 2016 Apr 9;387(10027):1561-72.

[6] Reinares M, Vieta E, Colom F, et al. Impact of a psychoeducational family intervention on caregivers of stabilized bipolar patients. Psychother Psycho- somat 2004;73:312-9.

[7] Miklowitz D, Wisniewski S, Miyahara S, et al. Perceived criticism from family members as a predictor of the 1-year course of bipolar disorder. Psychiatry Res 2005;136:10111.

[8] Miklowitz D, Goldstein M, Nuechterlein K, et al. Family factors and the course of bipolar affective disorder. Arch Gen Psychiatry 1988;45:225-31. 
[9] Hendrick, S. (2002). Les émotions exprimées: Le point de la recherche et son apport à la théorie familiale systémique. Cahiers critiques de thérapie familiale et de pratiques de réseaux, no 29,(2), 139-166. doi:10.3917/ctf.029.0139.

[10] Miklowitz DJ, George EL, Axelson DA, et al. Family-focused treatment for adolescents with bipolar disorder. J Affect Disord. 2004 Oct;82 Suppl 1:S113-28.

[11] Belardinelli C, Hatch JP, Olvera RL, et al. Family environment patterns in families with bipolar children. J Affect Disord. 2008 Apr;107(1-3):299-305.

[12] Jauhar S, McKenna PJ, Laws KR. Psychosocial treatments in bipolar disorder. Lancet Psychiatry. 2016 Apr;3(4):321.

[13] Rapport de l'OMS-Europe, publié en 1996, Therapeutic Patient Éducation Continuing Éducation Programmes for Health Care Providers in the field of Chronic Disease, traduit en français en 1998

[14] World Health Organization, Centre for Health Development. A glossary of terms for community health care and services for older persons. Kobe: WHO; 2004

[15] World Health Organization. Skills for health. Geneva : WHO ; 2003

[16] World Health Organization, Centre for Health Development. A glossary of terms for community health care and services for older persons. Kobe: WHO; 2004

[17] de Barros Pellegrinelli K, de O Costa LF, Silval KI, et al. Efficacy of psychoeducation on symptomatic and functional recovery in bipolar disorder. Acta Psychiatr Scand. 2013 Feb;127(2):153-8.

[18] Castle DJ, Berk M, Hocking BM. Bipolar disorder: new understandings, emerging treatments. Med J Aust. 2010 Aug 16;193(4 Suppl):S3-4.

[19] Colom F, Vieta E, Martinez-Aran A, et al. A randomized trial on the efficacy of group psychoeducation in the prophylaxis of recurrences in bipolar patients whose disease is in remission. Arch Gen Psychiatry. 2003 Apr;60(4):402-7.

[20] Colom F, Vieta E, Sánchez-Moreno J, et al. Group psychoeducation for stabilised bipolar disorders: 5-year outcome of a randomised clinical trial. Br J Psychiatry. 2009 Mar;194(3):260-5.

[21] D'Souza R, Piskulic D, Sundram S. A brief dyadic group based psychoeducation program improves relapse rates in recently remitted bipolar disorder: a pilot randomised controlled trial. J Affect Disord 2010; 120: 272-6.

[22] Bond K, Anderson IM. Psychoeducation for relapse prevention in bipolar disorder: a systematic review of efficacy in randomized controlled trials. Bipolar Disord. 2015 Jun;17(4):349-62.

[23] Eker F, Harkın S. Effectiveness of six-week psychoeducation program on adherence of patients with bipolar affective disorder. J Affect Disord. 2012 May;138(3):409-16.

[24] Etain B, Scott J, Cochet B, et al. A study of the real-world effectiveness of group psychoeducation for bipolar disorders: Is change in illness perception a key mediator of benefit? J Affect Disord. 2018 Feb;227:713-720.

[25] Colom F, Vieta E, Reinares M, et al. Psychoeducation efficacy in bipolar disorders: beyond compliance enhancement. J Clin Psychiatry. 2003 Sep;64(9):1101-5.

[26] Colom F, Vieta E, Sánchez-Moreno J, et al. Psychoeducation for bipolar II disorder: an exploratory, 5-year outcome subanalysis. J Affect Disord. 2009 Jan;112(1-3):30-5.

[27] Colom F, Vieta E, Sánchez-Moreno J, et al. Psychoeducation in bipolar patients with comorbid personality disorders. Bipolar Disord. 2004 Aug;6(4):294-8.

[28] Miller IW, Solomon DA, Ryan CE, et al. Does adjunctive family therapy enhance recovery from bipolar I mood episodes? J Affect Disord 2004; 82: 431-6.

[29] Bordbar MRF. Short-term family-focused psycho-educational program for bipolar 
mood disorder in Mashhad. Iran J Med Sci 2009; 34: 104-9.

[30] Madigan K, Egan P, Brennan D, et al. ? A randomised controlled trial of carerfocussed multi-family group psychoeducation in bipolar disorder. Eur Psychiatry 2012; 27: 281-4.

[31] Perlick DA, Miklowitz DJ, Lopez N, et al. Family-focused treatment for caregivers of patients with bipolar disorder. Bipolar Disord 2010; 12: 627-37.

[32] Reinares M, Colom F, Sanchez-Moreno J, et al. Impact of caregiver group psychoeducation on the course and outcome of bipolar patients in remission: a randomized controlled trial. Bipolar Disord 2008; 10: 511-9.

[33] Van Gent EM, Zwart FM. Psychoeducation of partners of bipolar-manic patients. J Affect Disord 1991; 21: 15-8.

[34] Reinares M, Colom F, Martínez-Arán A, et al. Therapeutic interventions focused on the family of bipolar patients. Psychother Psychosom. 2002 Jan-Feb;71(1):2-10. Review.

[35] Reinares M, Vieta E, Colom F, et al. What really matters to bipolar patients' caregivers: sources of family burden. J Affect Disord 2006;94:157-63.

[36] Goldstein MJ, Miklowitz DJ. Family intervention for persons with bipolar disorder. New Dir Ment Health Serv. 1994 Summer;(62):23-35. PubMed PMID: 7935284.

[37] Miklowitz DJ, Simoneau TL, George EL, et al. Family-focused treatment of bipolar disorder: 1-year effects of a psychoeducational program in conjunction with pharmacotherapy. Biol Psychiatry 2000; 48: 582-92.

[38] Miklowitz DJ, Axelson DA, Birmaher B, et al. Family-focused treatment for adolescents with bipolar disorder: results of a 2-year randomized trial. Arch Gen Psychiatry. 2008 Sep;65(9):1053-61.

[39] Tompson MC, Rea MM, Goldstein MJ, et al. Difficulty in implementing a family intervention for bipolar disorder: the predictive role of patient and family attributes. Fam Process. 2000 Spring;39(1):105-20.

[40] Oud M, Mayo-Wilson E, Braidwood R, et al. Psychological interventions for adults with bipolar disorder: systematic review and meta-analysis. Br J Psychiatry. 2016 Mar;208(3):213-22.

[41] Lacroix A. L'éducation thérapeutique des patients, nouvelles approches de la maladie. Maloine; 2003.

[42] Derguy C. L'ajustement parental dans les troubles du spectre de l'autisme. Etude des facteurs de protection et de vulnérabilité et développement d'un programme d'éducation thérapeutique (these) Bordeaux: Université de Bordeax; 2014

[43] Colom F, Vieta E, Martínez A, et al. What is the role of psychotherapy in the treatment of bipolar disorder? Psychother Psychosom. 1998;67(1):3-9. Review.

[44] Haute Autorité de Santé. La prise en charge d'un trouble bipolaire. Vivre avec un trouble bipolaire. 2010. Decembre. Guide affection de longue durée

[45] Ausloos G. La compétence des familles: temps, chaos, processus. Erès; 1995.

[46] Cook-Darzens S. Thérapies multifamiliales: des groupes comme agents thérapeutiques. Eres; 2007.

[47] Favez N. L'examen clinique de la famille: modèles et instruments d'évaluation. Vol. 5. Editions Mardaga; 2011.

[48] Moss-Morris R, Weinman J, Petrie $\mathrm{K}$, et al. The revised illness perception questionnaire (IPQ-R). Psychology and health $2002 ; 17(1), 1-16$.

[49] d'Ivernois JF, \& Gagnayre R. Compétences d'adaptation à la maladie du patient: une proposition. Éducation thérapeutique du patient-Therapeutic patient education $2011 ; 3(2)$, S201-S205. 
[50] Loi, H. P. S. T. Loi n 2009-879 du 21 juillet 2009 portant réforme de l'hôpital et relative aux patients, à la santé et aux territoires. Legifrance. gouv. fr, $2009 ; 21$.

[51] Foucaud J, Bury JA, Balcou-Debussche M, et al. Éducation thérapeutique du patient. Modèles, pratiques et évaluation. hal.archives-ouvertes.fr; 2010.

[52] M'Bailara K, \& Gay C. Famille et troubles bipolaires. In Les troubles bipolaires. Lavoisier 2014 ; pp. 541-550.

[53] Kim EY, Miklowitz DJ. Expressed emotion as a predictor of outcome among bipolar patients undergoing family therapy. J Affect Disord. 2004 Nov 1;82(3):343-52.

[54] Carr A. Bipolar disorder in young people: Description, assessment and evidencebased treatment. Dev Neurorehabil. 2009;12(6):427-41.

[55] Fristad MA, Goldberg-Arnold JS, Gavazzi SM. Multifamily psychoeducation groups (MFPG) for families of children with bipolar disorder. Bipolar Disord. 2002 Aug;4(4):254-62.

[56] Miklowitz DJ, Scott J. Psychosocial treatments for bipolar disorder: costeffectiveness, mediating mechanisms, and future directions. Bipolar Disord. 2009 Jun;11 Suppl 2:110-22. 


\begin{tabular}{|c|c|c|c|}
\hline & Avant l'ETP & Après l'ETP & Différence \\
\hline Modification des connaissances & $12.34($ SD 4.46$)$ & 15.7 (SD 3.42) & $\mathrm{V}=1917.5, \mathrm{p}$-value $=3.21 \mathrm{e}-08^{* * *}$ \\
\hline Modification de la perception du trouble & $51.42($ SD 9.92) & 41.14 (SD 15.13) & $\mathrm{V}=340.5, \mathrm{p}$-value $=7.849 \mathrm{e}-08^{* * *}$ \\
\hline Diminution score conscience & 6.58 (SD 2.46) & $6.01($ SD 2.74) & $V=584.5, p$-value $=0.07807$ \\
\hline Score chronicité & 8.13 (SD 2.25) & 8.69 (SD 2.26) & $V=565, p$-value $=0.07926$ \\
\hline Score manque de contrôle & $5.23(\mathrm{SD} 2.42)$ & 4.21 (SD 2.43) & $\mathrm{V}=405.5, \mathrm{p}$-value $=0.0004271^{* * *}$ \\
\hline Doute sur le traitement & $3.3(\mathrm{SD} 2.38)$ & $2.31(\mathrm{SD} 2.26)$ & $V=362, p$-value $=0.0005562^{* * *}$ \\
\hline Diminution score sévérité du trouble & $5.12(\mathrm{SD} 2.12)$ & 4.78 (SD 2.52) & $\mathrm{V}=729.5, \mathrm{p}$-value $=0.2367$ \\
\hline Diminution du score d'intensité de l'inquiétude & 6.47 (SD 2.34) & 5.85 (SD 2.53) & $V=350, p$-value $=0.00831^{* *}$ \\
\hline Diminution du score d'incompréhension & 4.67 (SD 2.25) & 2.58 (SD 1.82) & $V=91, p-$ value $=1.662 \mathrm{e}-09^{* * *}$ \\
\hline Diminution du score d'impact émotionnel & 6.96 (SD 2.33) & 6.19 (SD 2.72) & $\mathrm{V}=416, \mathrm{p}$-value $=0.002783^{* * *}$ \\
\hline Diminution du score d'accord avec le diagnostic & 5.54 (SD 3.56) & 2.17 (SD 2.55) & $\mathrm{V}=198, \mathrm{p}$-value $=5.72 \mathrm{e}-07^{* * *}$ \\
\hline
\end{tabular}

Note. $^{* * *}: \mathrm{p}<.01 ;$ Note. ${ }^{* *}: \mathrm{p}<.05 ;^{*}: \mathrm{p}<.10$

Tableau 3. Modification de perception du trouble après 12 séances d’Education thérapeutique chez des patients ayant un trouble bipolaire 


\begin{tabular}{|c|c|c|c|}
\hline & Avant l'ETP & Après l'ETP & Différence \\
\hline Modification des connaissances & $12.34($ SD 4.46$)$ & 15.7 (SD 3.42) & $\mathrm{V}=1917.5, \mathrm{p}$-value $=3.21 \mathrm{e}-08^{* * *}$ \\
\hline Modification de la perception du trouble & $51.42($ SD 9.92) & 41.14 (SD 15.13) & $\mathrm{V}=340.5, \mathrm{p}$-value $=7.849 \mathrm{e}-08^{* * *}$ \\
\hline Diminution score conscience & 6.58 (SD 2.46) & $6.01($ SD 2.74) & $V=584.5, p$-value $=0.07807$ \\
\hline Score chronicité & 8.13 (SD 2.25) & 8.69 (SD 2.26) & $V=565, p$-value $=0.07926$ \\
\hline Score manque de contrôle & $5.23(\mathrm{SD} 2.42)$ & 4.21 (SD 2.43) & $\mathrm{V}=405.5, \mathrm{p}$-value $=0.0004271^{* * *}$ \\
\hline Doute sur le traitement & $3.3(\mathrm{SD} 2.38)$ & $2.31(\mathrm{SD} 2.26)$ & $V=362, p$-value $=0.0005562^{* * *}$ \\
\hline Diminution score sévérité du trouble & $5.12(\mathrm{SD} 2.12)$ & 4.78 (SD 2.52) & $\mathrm{V}=729.5, \mathrm{p}$-value $=0.2367$ \\
\hline Diminution du score d'intensité de l'inquiétude & 6.47 (SD 2.34) & 5.85 (SD 2.53) & $V=350, p$-value $=0.00831^{* *}$ \\
\hline Diminution du score d'incompréhension & 4.67 (SD 2.25) & 2.58 (SD 1.82) & $V=91, p-$ value $=1.662 \mathrm{e}-09^{* * *}$ \\
\hline Diminution du score d'impact émotionnel & 6.96 (SD 2.33) & 6.19 (SD 2.72) & $\mathrm{V}=416, \mathrm{p}$-value $=0.002783^{* * *}$ \\
\hline Diminution du score d'accord avec le diagnostic & 5.54 (SD 3.56) & 2.17 (SD 2.55) & $\mathrm{V}=198, \mathrm{p}$-value $=5.72 \mathrm{e}-07^{* * *}$ \\
\hline
\end{tabular}

Note. $^{* * *}: \mathrm{p}<.01 ;$ Note. ${ }^{* *}: \mathrm{p}<.05 ;^{*}: \mathrm{p}<.10$

Tableau 3. Modification de perception du trouble après 12 séances d’Education thérapeutique chez des patients ayant un trouble bipolaire 


\begin{tabular}{|c|c|c|c|}
\hline & Avant l'ETP & Après l'ETP & Différence \\
\hline Modification des connaissances & $12.34($ SD 4.46$)$ & 15.7 (SD 3.42) & $\mathrm{V}=1917.5, \mathrm{p}$-value $=3.21 \mathrm{e}-08^{* * *}$ \\
\hline Modification de la perception du trouble & $51.42($ SD 9.92) & 41.14 (SD 15.13) & $\mathrm{V}=340.5, \mathrm{p}$-value $=7.849 \mathrm{e}-08^{* * *}$ \\
\hline Diminution score conscience & 6.58 (SD 2.46) & $6.01($ SD 2.74) & $V=584.5, p$-value $=0.07807$ \\
\hline Score chronicité & 8.13 (SD 2.25) & 8.69 (SD 2.26) & $V=565, p$-value $=0.07926$ \\
\hline Score manque de contrôle & $5.23(\mathrm{SD} 2.42)$ & 4.21 (SD 2.43) & $\mathrm{V}=405.5, \mathrm{p}$-value $=0.0004271^{* * *}$ \\
\hline Doute sur le traitement & $3.3(\mathrm{SD} 2.38)$ & $2.31(\mathrm{SD} 2.26)$ & $V=362, p$-value $=0.0005562^{* * *}$ \\
\hline Diminution score sévérité du trouble & $5.12(\mathrm{SD} 2.12)$ & 4.78 (SD 2.52) & $\mathrm{V}=729.5, \mathrm{p}$-value $=0.2367$ \\
\hline Diminution du score d'intensité de l'inquiétude & 6.47 (SD 2.34) & 5.85 (SD 2.53) & $V=350, p$-value $=0.00831^{* *}$ \\
\hline Diminution du score d'incompréhension & 4.67 (SD 2.25) & 2.58 (SD 1.82) & $V=91, p-$ value $=1.662 \mathrm{e}-09^{* * *}$ \\
\hline Diminution du score d'impact émotionnel & 6.96 (SD 2.33) & 6.19 (SD 2.72) & $\mathrm{V}=416, \mathrm{p}$-value $=0.002783^{* * *}$ \\
\hline Diminution du score d'accord avec le diagnostic & 5.54 (SD 3.56) & 2.17 (SD 2.55) & $\mathrm{V}=198, \mathrm{p}$-value $=5.72 \mathrm{e}-07^{* * *}$ \\
\hline
\end{tabular}

Note. $^{* * *}: \mathrm{p}<.01 ;$ Note. ${ }^{* *}: \mathrm{p}<.05 ;^{*}: \mathrm{p}<.10$

Tableau 3. Modification de perception du trouble après 12 séances d’Education thérapeutique chez des patients ayant un trouble bipolaire 